\title{
An alternative approach for the management of missing lateral incisors through the intentional retention of primary canines
}

\author{
Eun-Jeong Jang, Tae-Sung Jeong, Jong-Hyun Shin, and Shin Kim* \\ Department of Pediatric Dentistry, Pusan National University School of Dentistry, Yangsan, Republic of Korea
}

\begin{abstract}
Patients with congenitally missing or undeveloped lateral incisors are less likely to have normal teeth alignment. Traditional treatment methods in these patients have been used for creating space for prosthetic treatment or close space using orthodontic treatment. A novel approach comprising the intentional retention of the primary canine followed by the positioning of the central incisor, permanent canine, primary canine, and first premolar in alignment may be considered. Using proper diagnosis, timely interventions, and orthodontic and prosthetic consultations, the primary canines could be retained in the dentition, thus maintaining the occlusal relationship and achieving aesthetic teeth alignment. Intentional preservation of primary canines may be a useful alternative for treating adolescents with agenesis of the lateral incisors.
\end{abstract}

Key Words: Congenital missing, Lateral incisors, Retained tooth

cc This is an open-access article distributed under the terms of the Creative Commons Attribution Non-Commercial License (http://creativecommons.org/licenses/by-nc/4.0) which permits unrestricted noncommercial use, distribution, and reproduction in any medium, provided the original work is properly cited.

\section{INTRODUCTION}

Tooth agenesis is a relatively common problem, with a prevalence of $2.5 \%-6.9 \%$. In particular, the prevalence of upper lateral incisor agenesis is $1.6 \%-1.8 \%$, the second highest after that of lower 2nd premolar agenesis [1,2]. In addition, early extraction of the lateral incisors may be performed due to a lack of tooth development.

Traditional treatment methods have included the maintenance of prosthetic spaces and the cautious closure of the spaces until adult prosthetic treatment could be performed; both methods have distinct advantages and disadvantages.

When the prosthetic space is maintained, a space maintainer will be worn until future prosthetic treatment includ- ing implant could be performed. However, the coordination of the child may be reduced and the risk of caries will be increased by prolonged wearing of a space maintainer. In addition, the alveolar ridge would be gradually thinned due to tooth loss, which causes difficulty in future prosthetic procedures [3].

An alternative treatment option is the substitution of a canine for the missing lateral incisor, through orthodontic treatment. This method might enable avoidance of prosthetic treatment, but could involve difficulty when applied to non-crowded dentition [4]. In addition, ideal occlusion might be difficult to achieve, and aesthetic limitations might be encountered because of differences in morphology, color, root volume, and torque between the lateral

Received November 11, 2019; Revised November 14, 2019; Accepted November 14, 2019

*Corresponding author: Shin Kim, Department of Pediatric Dentistry, Pusan National University School of Dentistry, 20 Geumo-ro, Mulgeumeup, Yangsan 50612, Republic of Korea.

Tel: +82-55-360-5173, Fax: +82-55-360-5174, E-mail: shinkim@pusan.ac.kr 
incisor and canine [5].

When agenesis of the lateral incisor could be diagnosed before the onset of natural root resorption of the primary canine, clinicians might consider alternative treatments that enable intentional preservation of the primary canine with intact roots. Among primary teeth retained in the adult dentition, primary canines are reported to have the longest life expectancy in the oral cavity, similar to that of primary 2nd molars [6]. In a previously reported case, an upper primary canine was maintained in the adult dentition for more than 50 years [7].

In this case report, the anterior space was managed to guide the permanent canine into the lateral incisor space. The intention was to use the primary canine to retain the positions of the central incisor, permanent canine, primary canine, and 1st premolar. In addition, the feasibility, significance, and limitations of this new technique were examined.

\section{CASE}

\section{Case 1}

A 6-year-old girl was diagnosed with congenital agenesis of both maxillary lateral incisors. After 1 year, the central incisors had erupted, with the eruption path of both permanent canines set to the mesial side (Fig. 1A). A treatment plan was designed to allow both maxillary canines to erupt into the lateral incisor positions. A rapid palatal expansion (RPE) appliance was attached for prevention of root resorption of primary canines. RPE expanded once a day. The total number of expansions was 28 and the screw was expanded $7 \mathrm{~mm}$. Orthodontic brackets were applied to the central incisors for the purpose of rearranging space for canine eruptions. After 1 year and 4 months the RPE was removed and treatment terminated. After 2 years and 3 months, both maxillary canines were induced to move to the lateral incisor spaces and both primary canines were retained without root resorption (Fig. 1B, C).
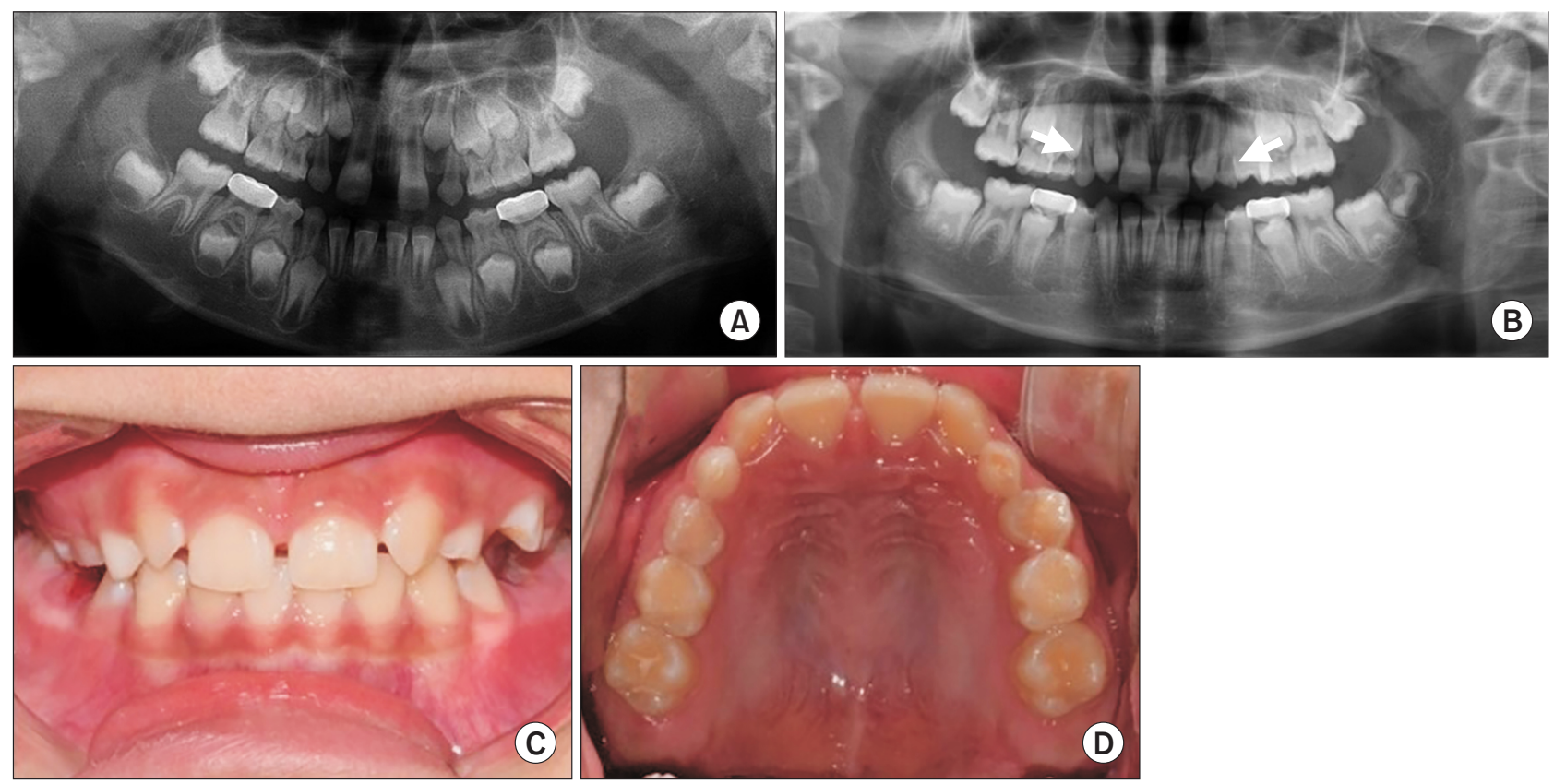

Fig. 1. (A) Initial panoramic radiograph with agenesis of both maxillary lateral incisors. (B) Panoramic radiograph and (C, D) intraoral photographs 4 years after the start of treatment. 


\section{Case 2}

A 8-year-old boy was visited the pediatric dentistry department for fusion of primary maxillary left incisors. Radiographic examination revealed that maxillary left lateral incisor was an underdeveloped microdontia and normal eruption was seemed difficult (Fig. 2A). The treatment plan involved surgical removal of the tooth bud of the maxillary left lateral incisor and guidance of the permanent canine to erupt into the lateral incisor position. An RPE appliance was used to expand the maxilla for 4 weeks. RPE expanded once a day. The RPE was expanded a total of 28 times and the screw was expanded $7 \mathrm{~mm}$. The guidewire reached the distal surface of the central incisor and was connected to the opposite section of the RPE device. A guidewire was used to prevent the central incisor from moving into the distal position while the midline suture being expanded. As a result, diastema was not occurred and the midline remained aesthetic. A labial bow was added to prevent labioversion of the maxillary anterior teeth during palatal expansion (Fig. 2C, D). After 6 months of expansion completion, the root of the maxillary left primary canine was able to remain intact without resorption, and the permanent ca- nine was successfully moved to the lateral incisor position (Fig. 2B). Periodic follow-up is expected to be necessary until permanent canine eruption could be achieved.

\section{Case 3}

A 6-year-old boy visited the pediatric dentistry department with the chief complaint of congenital agenesis of both mandibular lateral incisors. On the initial panorama, the permanent mandibular right canine was located directly below primary canine, and the permanent mandibular left canine tended to be inclined toward the mesial side (Fig. $3 \mathrm{~A}$. Compared to the right side where the primary mandibular right lateral incisor was lost, the left side, primary left lateral incisor was remained and considered to be able to induce eruption of the permanent canine into the incisal position. Therefore, primary mandibular left lateral incisor was intentionally extracted. During 4 years and 8 months of follow up, permanent canine was found to erupt into the lateral incisor site. Any sign of root resorption of the primary left canines was not found. On the right, the primary canine is normally exchanged for permanent canine (Fig. 3BD). At the age of 10 years and 8 months, all primary tooth
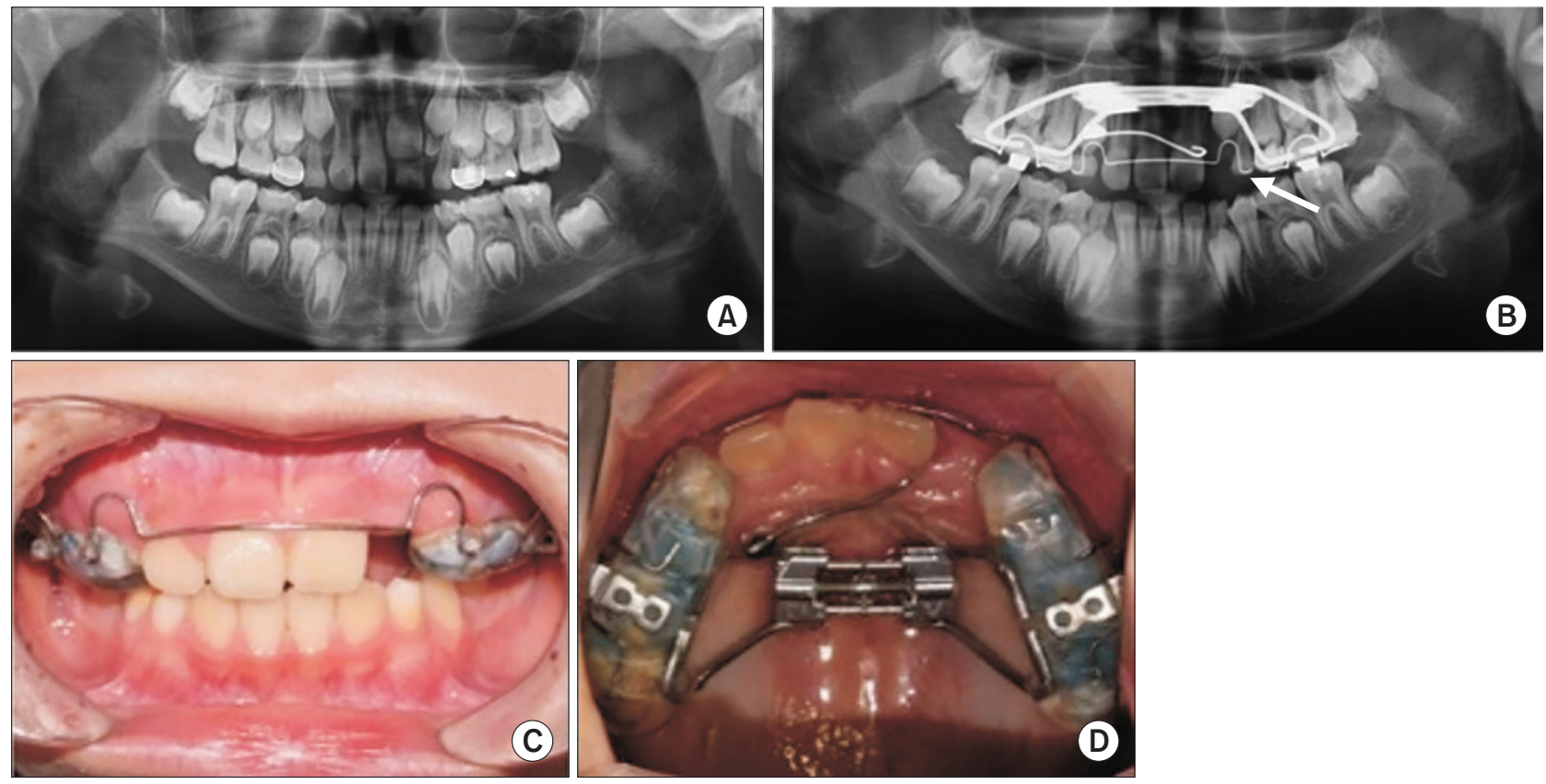

Fig. 2. (A) Initial panoramic radiograph with microdontia and underdevelopment of the maxillary left lateral incisor. (B) Panoramic radiograph taken 1 year after the start of treatment. (C, D) Intraoral photographs of rapid palatal expansion with guide wire and labial bow. 

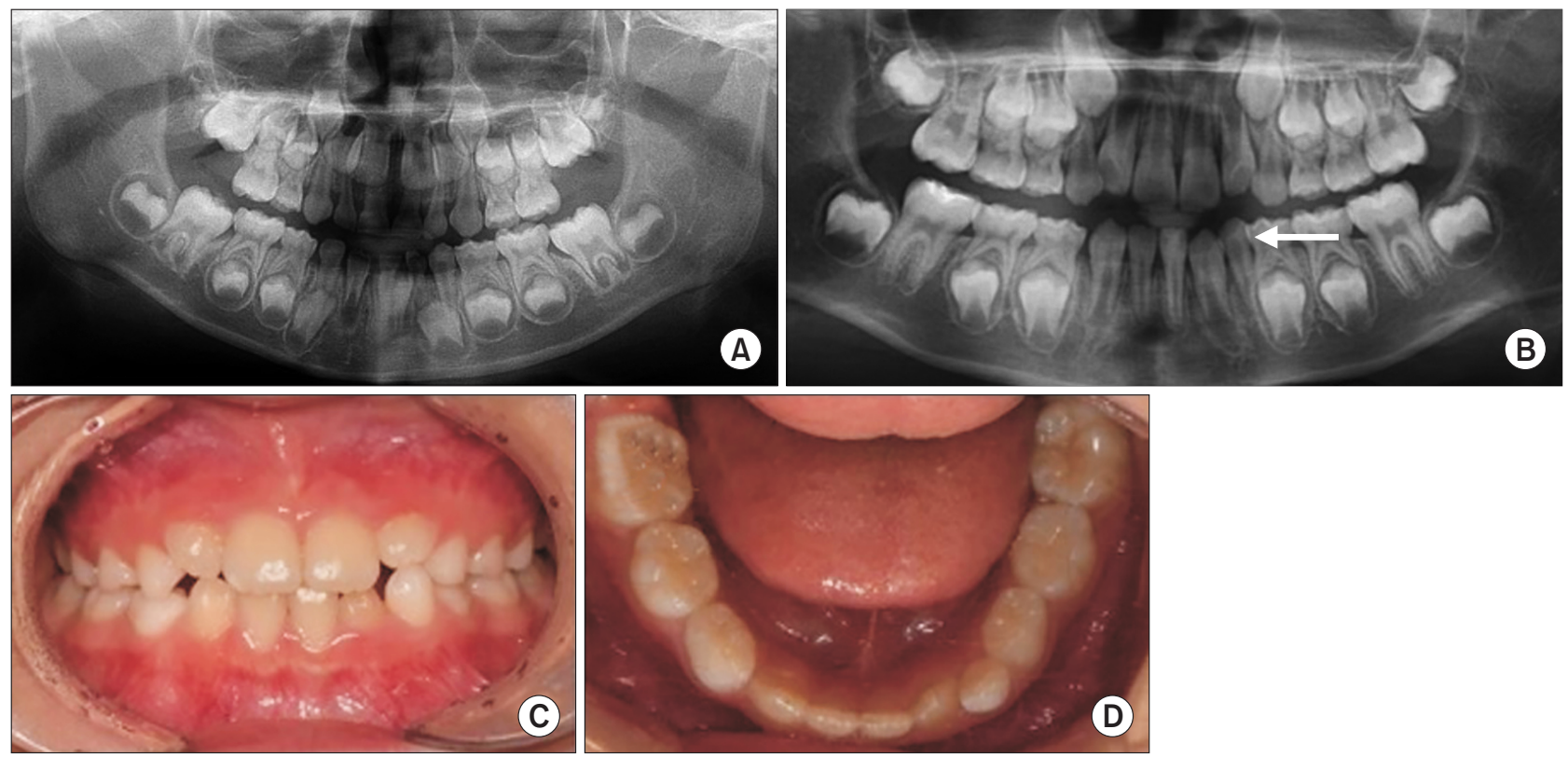

Fig. 3. (A) Initial panoramic radiograph with congenital agenesis of both mandibular lateral incisors. (B) Panoramic radiograph taken 4 years 8 months after initial oral exam. (C, D) Primary left canine was retained while primary right canine exfoliated.

except the primary mandibular left canine were exfoliated and consultation with the orthodontist was conducted to establish a long-term treatment plan. In the orthodontic evaluation, it was found that additional orthodontic treatment was unnecessary because the root of primary canine was intact and the occlusion was stable.

\section{DISCUSSION}

In this report, a technique was described for intentional preservation of the primary canine and substitution of a permanent canine for a lost permanent lateral incisor. For application of this method, case selection is considered to be important. The subjects of this treatment should be patients in whom extraction treatment cannot be performed due to crowding. In patients with severe space deficiency, the consideration of orthodontic space closure may be advantageous [8]. However, for patients without crowding or other orthodontic problems, it is hard to achieve ideal occlusion through orthodontic space closure in extraction treatment. These kind of patients are the subjects of this treatment.

In order to achieve more stable occlusion through orthodontic treatment, it is ideal to form functional normal oc- clusion through compensatory extraction of left and right, upper and lower dentition. In cases without crowding as in case 2, compensatory extraction for missing teeth has disadvantages in terms of space closure and tooth preservation. Without compensatory extraction, it would be difficult to obtain ideal occlusal relationship of molars. Therefore, in case 2, it would be more advantageous to perform expansion treatment to form more ideal occlusion.

When considering intentional preservation of the primary canine, a diagnosis of agenesis or underdevelopment of the lateral incisors should be established early, before the root of the primary canine is naturally resorbed. Many studies of the timing of primary canine resorption have been conducted. In a study by Haavikko [9], it was reported that resorption of the primary canine began when one-quarter of the root of successor had formed. In a study of children' s skulls conducted by Saka et al. [10], root resorption was observed from the lingual side of the primary canine root when the central incisors had reached the alveolar crest. In a study conducted by Daito et al. [11], it was found that the mean ages of resorption of the upper primary canine were 6.9 years for girls and 7.1 years for boys, whereas the mean ages of resorption of the lower primary canine were 6.3 years for girls and 6.9 years for boys. Periodic clinical and 
radiographic examinations should be performed to identify adequate therapeutic interventions for prognostic improvement.

The prevention of root resorption of the primary canine during treatment is an important issue. The roots are resorbed as the eruption cyst of the permanent tooth approaches. Therefore, sufficient space must be secured to prevent the resorption of the primary canine root during guidance of the permanent canine to the lateral incisor site. Mid-palatal sutures are expanded into fan shapes by using an RPE device, which is effective for the creation of space in the premaxilla [12]. In this case report, maintenance of the primary canine was achieved by maxillary expansion using an RPE appliance.

In the case 3, patient had congenital missing of both permanent mandibular lateral incisors. The aim was to arrange 2 central incisors and 1 permanent canine in the 4 anterior tooth spaces, so expansion was not necessary. Extraction of the primary tooth promotes the eruption of permanent teeth, and the effect of promoting eruption is better when the tooth growth of the permanent tooth is not completed [13]. Therefore, intentional early extraction of the primary lateral incisor was performed, which induced mesial eruption of permanent canine by reducing resistance of eruption pathway.

However, variation in the success rate might be observed, depending on the location of the permanent canine. In the third case reported here, the mandibular left canine tooth germ was located mesial to its normal position, which was advantageous for guidance of the canine toward the lateral incisor position. However, the right canine was located directly below the primary canine, such that difficultly was encountered in changing its position without root resorption of the primary canine. Lack of space for the permanent canine had also made it difficult to preserve the primary canine.

In case 1 and 2 , it was necessary to arrange 4 teeth in the anterior part, even for aesthetic reasons. Considering the difference in size between the lateral incisor and canine, and the difference between the size of the primary and permanent canine, there may be no overall lack of space. However, the purpose of the use of RPE in this case is not to alleviate crowding, but to secure intraosseous space through the expansion of premaxilla so that the permanent canine is induced into the lateral incisor positon without resorption of primary canine as much as possible. According to a study by McNamara et al. [14], after 8 years of maxillary expansion treatment, only $80 \%$ of the expansion was effective and recurrence occurred after sufficient retention period. In this study, it was necessary to maintain the expanded maxilla until permanent canine was induced into the lateral incisor position and did not absorb the root of the primary canine. Therefore, the RPE was maintained until the height of contour of the permanent canine crown passed through the alveolar bone, which took about 1 year. Case 3 was a mandibular case, with low aesthetic demands. In addition, the maxilla is easy to expand, but the mandible is limited to osteogenic expansion. Therefore, 3 teeth including permanent canine were arranged in 4 anterior tooth spaces through intentional extraction of primary incisor, and functional occlusion was obtained.

The primary canine might be used to maintain alveolar bone volume until final prosthetic treatment, including implant placement, in adulthood. As the primary canine is known to function as natural space maintainer, the wearing of an artificial space maintainer for an extended period might be avoided. If not, the patient should continue to use the removable space maintainer until adulthood, when the implant could be treated, which would reduce the patient's quality of life and cause problems with caries management. Regular visits to the dental clinic during the late teens' years of life would also be burdensome for patients. A retained primary canine could be included in the final occlusion, contributing to effective mastication and aesthetic dentition. According to Nordquist and McNeill [15], the posterior group function created by the relocated permanent canine is similar to the canine-protected function and is not a source of periodontal problems.

After eruption of a permanent canine, use of a fixed appliance might be required for root paralleling, as well as inclusion of the retained primary canine. In a prior case report, orthodontic treatment was performed with a fixed appliance attached to the primary canine for 32 months; the primary canine was well maintained for 5 years after treatment and was able to serve as a permanent canine. No clinical or radiological problem was observed following lat- 
eral guidance [16]. When orthodontic force is applied, root resorption may be caused by stress focused on the periodontal ligament. The mechanism is similar in permanent and primary teeth [17]. However, minimal data comparing root resorption between permanent and primary teeth are available [3]; thus, further research is needed.

To create a more aesthetic appearance of the dentition, restorative or prosthetic treatments could be considered for permanent and primary canines. The permanent canine is larger than the lateral incisor, and the labial surface is more convex; thus, significant amounts of tooth preparation and restoration are required [4]. Direct restoration with composite resin or indirect restoration like laminate veneer might be considered. When restoring primary teeth, the poor bond strength of the enamel of primary teeth should be considered [18]. Despite the availability of various treatment methods, aesthetic limitations might be in play, and should be explained to the patient in advance.

Accurate prediction of the long-term prognosis of a retained primary canine after the completion of growth is difficult, and the long-term stability of such teeth is uncertain. Shimizu et al. [19] found good occlusion of the primary canine serving as a permanent canine, through molar group function; fewer atrophic changes were observed in the periodontal ligament and alveolar bone. In a study conducted by Sletten et al. [20], retained primary teeth were found to remain healthy for several years under atraumatic and good occlusion. Conversely, in a study conducted by Aisenberg [21], the roots of primary teeth remaining in the dental arch of an adult were gradually resorbed under the occlusal force of the adult teeth. However, retained primary canines have been found to exhibit their own significance, at least until prosthetic treatment in early adulthood. If the primary canine is exfoliated after growth completion, space can be restored by implant prosthodontics combined with orthodontic treatment. It is meaningful to carry out this treatment only if the alveolar bone volume is sufficiently preserved. This approach may be considered a third method for avoidance of the limitations of traditional treatment for the loss of permanent lateral incisors.

The retention of primary canines could enable the effective maintenance of alveolar bone volume and prevention of space loss, thereby facilitating avoidance of long-term space maintainer use. Retained primary canines included in the final occlusion can help to achieve aesthetic dentition. By proper diagnosis, timely intervention, and orthodontic and prosthetic consultation, primary canines could be retained properly in the dentition, and an acceptable longterm prognosis might be expected. In adolescents with agenesis of the lateral incisors, intentional preservation of the primary canines by maxillary expansion and extraction of mandibular primary incisors might be a useful alternative treatment.

\section{ACKNOWLEDGEMENTS}

This paper was supported by Pusan National University in 2019.

\section{CONFLICTS OF INTEREST}

The authors declare that they have no competing interests.

\section{ORCID}

\author{
Eun-Jeong Jang \\ https://orcid.org/0000-0002-9796-568X \\ Tae-Sung Jeong \\ https://orcid.org/0000-0002-0431-5574 \\ Jong-Hyun Shin \\ https://orcid.org/0000-0002-9777-0196 \\ Shin Kim \\ https://orcid.org/0000-0002-7202-7726
}

\section{REFERENCES}

1. Polder BJ, Van't Hof MA, Van der Linden FP, Kuijpers-Jagtman AM. A meta-analysis of the prevalence of dental agenesis of permanent teeth. Community Dent Oral Epidemiol 2004;32:217-226. doi: 10.1111/j.1600-0528.2004.00158.x.

2. Krassnig M, Fickl S. Congenitally missing lateral incisors--a comparison between restorative, implant, and orthodontic approaches. Dent Clin North Am 2011;55:283-299, viii. doi: 10.1016/j.cden.2011.01.004.

3. Kenworthy CR, Larson BE. Incorporating retained deciduous teeth in orthodontic therapy. Am J Orthod Dentofacial Orthop 2001;119:202-210. doi: 10.1067/ 
mod.2001.112445.

4. Kokich VO Jr, Kinzer GA. Managing congenitally missing lateral incisors. Part I: canine substitution. J Esthet Restor Dent 2005;17:5-10. doi: 10.1111/j.1708-8240.2005. tb00076.x.

5. Silveira GS, de Almeida NV, Pereira DM, Mattos CT, Mucha JN. Prosthetic replacement vs space closure for maxillary lateral incisor agenesis: a systematic review. Am J Orthod Dentofacial Orthop 2016;150:228-237. doi: 10.1016/ j.ajodo.2016.01.018.

6. Haselden K, Hobkirk JA, Goodman JR, Jones SP, Hemmings $\mathrm{KW}$. Root resorption in retained deciduous canine and molar teeth without permanent successors in patients with severe hypodontia. Int J Paediatr Dent 2001;11:171-178. doi: 10.1046/j.1365-263x.2001.00257.x.

7. Stanley HR, Collett WK, Hazard JA. Retention of a maxillary primary canine: fifty years above and beyond the call of duty. ASDC J Dent Child 1996;63:123-130.

8. Tuverson DL. Orthodontic treatment using canines in place of missing maxillary lateral incisors. Am J Orthod 1970;58:109-127. doi: 10.1016/0002-9416(70)90065-5.

9. Haavikko K. Correlation between the root resorption of deciduous teeth and the formation of the corresponding permanent teeth. Proc Finn Dent Soc 1973;69:191-201.

10. Saka H, Koyama T, Tamatsu Y, Usami A, Ide Y. The morphological studies of root resorption of maxillary primary canines and their relation with the position of successive permanent teeth using Micro-CT. Pediatr Dent J 2011;21:145-153. doi: 10.1016/S0917-2394(11)70241-5.

11. Daito M, Kawahara S, Kato M, Okamoto K, Imai G, Hieda T. Radiographic observations on root resorption in the primary dentition. J Osaka Dent Univ 1991;25:1-23. doi: 10.18905/jodu.25.1_1.

12. Ghoneima A, Abdel-Fattah E, Hartsfield J, El-Bedwehi A, Kamel A, Kula K. Effects of rapid maxillary expansion on the cranial and circummaxillary sutures. Am J Orthod Dentofacial Orthop 2011;140:510-519. doi: 10.1016/ j.ajodo.2010.10.024.

13. Korean Academy of Pediatric Dentistry. Textbook of pediatric dentistry. 5th ed. Seoul: Yenang Inc; 2014;541.

14. McNamara JA Jr, Baccetti T, Franchi L, Herberger TA. Rapid maxillary expansion followed by fixed appliances: a long-term evaluation of changes in arch dimensions. Angle Orthod 2003;73:344-353. doi: 10.1043/0003-3219(2003)073〈0344:RMEFBF〉2.0.CO;2.

15. Nordquist GG, McNeill RW. Orthodontic vs. restorative treatment of the congenitally absent lateral incisor--long term periodontal and occlusal evaluation. J Periodontol 1975;46:139-143. doi: 10.1902/jop.1975.46.3.139.

16. Hwang S, Choi YJ, Chung CJ, Kim KH. Long-term survival of retained deciduous mandibular second molars and maxillary canine incorporated into final occlusion. Korean J Orthod 2017; 47:323-333. doi: 10.4041/kjod.2017.47.5.323.

17. Consolaro A. Orthodontic movement in deciduous teeth. Dental Press J Orthod 2015;20:16-19. doi: 10.1590/21769451.20.2.016-019.oin.

18. Endo T, Yoshino S, Shinkai K, Ozoe R, Shimada M, Katoh Y, Shimooka S. Shear bond strength differences of types of maxillary deciduous and permanent teeth used as anchor teeth. Angle Orthod 2007;77:537-541. doi: 10.2319/0003-3219(2007)077[0537:SBSDOT]2.0.CO;2.

19. Shimizu Y, Hosomichi J, Nakamura S, Ono T. Micro-computed tomography analysis of changes in the periodontal ligament and alveolar bone proper induced by occlusal hypofunction of rat molars. Korean J Orthod 2014;44:263267. doi: 10.4041/kjod.2014.44.5.263.

20. Sletten DW, Smith BM, Southard KA, Casko JS, Southard TE. Retained deciduous mandibular molars in adults: a radiographic study of long-term changes. Am J Orthod Dentofacial Orthop 2003;124:625-630. doi: 10.1016/ j.ajodo.2003.07.002.

21. Aisenberg MS. The tissues and changes involved in orthodontic tooth movements. Am J Orthod 1948;34:854-859. doi: 10.1016/0002-9416(48)90018-9. 